\title{
High pressure phase transitions for CdSe
}

\author{
BO KONG ${ }^{1,2, *}$, TI-XIAN ZENG ${ }^{3}$, ZHU-WEN ZHOU ${ }^{1}$, DE-LIANG CHEN ${ }^{1}$ and XIAO-WEI SUN ${ }^{4}$ \\ ${ }^{1}$ School of Physics and Electronic Sciences, Guizhou Normal College, Guiyang 550018, China \\ ${ }^{2}$ Guizhou Provincial Key Laboratory of Computational Nano-Material Science, Guizhou Normal College, \\ Guiyang 550018, China \\ ${ }^{3}$ College of Physics and Electronic Information, China West Normal University, Nanchong 637002, China \\ ${ }^{4}$ School of Mathematics and Physics, Lanzhou Jiaotong University, Lanzhou 730070, China
}

MS received 17 December 2012; revised 5 February 2013

\begin{abstract}
The structure and pressure-induced phase transitions for CdSe are investigated using firstprinciples calculations. The pressure-induced phase transition sequence $\mathrm{WZ} / \mathrm{ZB} \rightarrow \mathrm{Rs} \rightarrow \mathrm{Cmcm} \rightarrow \mathrm{CsCl}$ for CdSe is drawn reasonably for the fist time, the corresponding transition pressures are 3.8, 29 and $107 \mathrm{GPa}$, respectively and the intermediate states between the $\mathrm{Cm} \mathrm{cm}$ structure and the $\mathrm{CsCl}$ structure should exist.
\end{abstract}

Keywords. Semiconductor; high pressure; phase transition.

\section{Introduction}

CdSe has become quite interesting and important because of its major applications in solar cells and other optoelectronic devices due to its optimum bandgap $(\sim 1.9 \mathrm{eV})$, high absorption coefficient and n-type of conductivity. $\mathrm{CdSe}$, along with some additive in it, forms an important class of semiconductor materials, which finds applications in low cost devices such as LEDs, solar panels, photodetectors, lasers, gas sensors, thin film transistors, etc. (Ramaiah et al 2001; Hankare et al 2004). So, it is not surprising that a great deal of fundamental studies in experiments have been done on CdSe bulk crystals (Hodes et al 1987; He et al 2008), nanocrystals (Asami et al 2003; Chahboun et al 2009), especially on CdSe thin films (Bhuse 2005; Chen et al 2008; Mohamed et al 2011) in recent years; while in theory, most authors mainly focused on the studies of bulk crystals because of the limitations of calculations. These theoretical researches involved in the crystal structure, electronic band structure, dielectric function and vibrational properties of $\mathrm{CdSe}$ at zero temperature and zero pressure (Huang and Ching 1993; Xu and Ching 1993; Ouendadji et al 2011), the pressure dependences of bandgap and elastic constants (Deligoz et al 2006; Zhuravlev 2007; Sarasamak 2010) as well as the pressure-induced phase transitions (Payne et al 1992; Zakharov et al 1995; Cote et al 1997; Benkhettou et al 2004). However, some problems about the high pressure phase transitions of CdSe need to be further clarified. In experiments, the pressure-induced phase transition sequence $\mathrm{WZ} / \mathrm{ZB} \rightarrow \mathrm{Rs} \rightarrow \mathrm{Cmcm}$ for $\mathrm{CdSe}$ was

\footnotetext{
*Author for correspondence (kgzsfxy@ sina.cn)
}

observed at around 3 (Milman et al 2000) and $27 \mathrm{GPa}$ (Perdew et al 1996), respectively. In theory, Zakharov et al (1995) predicted firstly a high pressure phase transition from Rs to $\mathrm{CsCl}-$ type at about $95 \mathrm{GPa}$ using ab initio pseudopotential method within the local-density approximation. The transition sequence under pressure WZ/ZB $\rightarrow$ Rs $\rightarrow$ $\mathrm{Cmcm}$ for CdSe has also been successfully simulated using the similar method in 1997 (Cote et al 1997), the corresponding transition pressures are 2.5 and $29 \mathrm{G}$, respectively. Later in 2004, Benkhettou et al (2004) again investigated the high pressure phase transitions of $\mathrm{CdSe}$ using the full-potential linear muffin-tin-orbital method (FP-LMTO), they obtained the pressure-induced phase transition sequence $\mathrm{WZ} / \mathrm{ZB} \rightarrow \mathrm{Rs} \rightarrow \mathrm{CsCl} \rightarrow \mathrm{Cmcm}$ and the corresponding transition pressures were about 3, 15, $21 \mathrm{GPa}$, respectively. It is noted that the results from Zakharov et al (1995) and Benkhettou et al (2004) have very large difference, while the high pressure $\mathrm{CsCl}$ phase is not observed until now (Benkhettou et al 2004). Thus, further theoretical and experimental works are desired.

In the present work, the crystal structure, as well as the pressure-induced structural phase transitions of $\mathrm{CdSe}$ were investigated using first-principles calculations. The organization of this communication is as follows. In $\S 2$, we give the computational details. The results and detailed discussions are presented in $\S 3$. A short conclusion is drawn in $\S 4$.

\section{Theoretical methods}

\subsection{Total energy electronic structure calculations}

We calculated the electronic structures of CdSe using the plane-wave pseudopotential density functional theory 
method through the Cambridge Serial Total Energy Package (CASTEP) (Payne et al 1992; Milman et al 2000), together with the generalized gradient approximation (GGA-PBE) (Perdew et al 1996) for the exchangecorrelation function. The Kohn-Sham equation was solved by means of the ultrasoft pseudopotentials introduced by Vanderbilt (1990). Pseudo-atomic calculations were performed for $\mathrm{Cd} 4 d^{10} 5 s^{2}$ and $\mathrm{Se} 4 s^{2} 4 p^{4}$. Integration over the Brillouin-zone (BZ) was executed using $20 \times 20 \times 18$ and $20 \times 20 \times 20$ Monkhorst-Pack grids for hexagonal (WZ) and cubic (ZB) CdSe, respectively. The cutoff energy in the calculations was $500 \mathrm{eV}$. The convergence of the total energies within $10^{-6} \mathrm{eV} /$ atom was ensured with these parameters. A full optimization of the unit cell structure for each target external pressure was performed using the Broyden-Fletcher-Goldfarb-Shenno (BFGS) minimization technique (Pfrommer et al 1997), which can let one obtain the structural parameters, energies and enthalpies for one crystal at different hydrostatic pressures. The drawn $E(V)$ data were fitted to the BirchMurnaghan equation of states (Birch 1947) under the quasi-harmonic Debye model (Blanco et al 2004). The equilibrium Gibbs free energy and bulk modulus and the first pressure derivative of bulk modulus at different pressures and temperatures were extracted.

\section{Results and discussion}

\subsection{Ground state structure and thermal equation of state}

In the cubic zinc-blende (ZB) and hexagonal wurzite (WZ) phases, the calculated zero-pressure equilibrium lattice constants, equilibrium cell volume $V_{0}$, bulk modulus $B_{0}$ and its pressure derivative $B_{0}^{\prime}$ together with the available experimental (Madelung et al 1982; Bonello and Fernandez 1993) and theoretical data (Rabani 2002; Deligoz et al 2006; Zhuravlev 2007) are summarized in table 1. It is seen that the obtained equilibrium lattice constants and cell volumes with CASTEP code (GGA-PBE) are a little bigger than the experimental data so that the drawn bulk modulus are underestimated. This may be due to the fact that GGA calculations usually overestimate the structural parameters (Zaoui and Elhaj Hassan 2001).
The thermal EOS is a measurement of the relationship among pressure, volume, and temperature $(P-V-T)$. The inclusion of temperature makes it more important than $P$ $V E O S$. In figure 1, we illustrate the normalized cell volume dependence on pressure for the ZB structural CdSe from 0 to $10 \mathrm{GPa}$ at $0 \mathrm{~K}, 300,500$ and $800 \mathrm{~K}$. The temperature effect is considered through the quasi-harmonic Debye model (Blanco et al 2004). Unfortunately, there are no available experimental or theoretical data for the comparison. It is noted that the normalized cell volume $V / V_{0}$ decreases with increasing pressure at a given temperature, and is not sensitive to temperature especially at low pressure. At a given pressure, the normalized cell volume $V / V_{0}$ of the $\mathrm{ZB}$ structural $\mathrm{CdSe}$ also decreases with increasing temperature, which means it is easier to compress $\mathrm{CdSe}$ at high temperature. In fact, the $\mathrm{ZB}$ or $\mathrm{WZ}$ structural CdSe transforms to RS structure ( $\mathrm{NaCl}$ structure) around $3 \mathrm{GPa}$ in experiment ( $\mathrm{Yu}$ and Gielisse 1971); whether the ZB or WZ structural CdSe will transform to other structure with increasing temperature is unknown. In principle, one could obtain the phase diagram of one crystal material combining first-principle calculations and the quasi-harmonic Debye model since Gibbs free energy, at different pressures and temperatures for the different structural phases, could be extracted from the calculated $E-V$ data points. However, there are almost no reports about the successful examples for our best knowledge. This is the serious limitation of the quasiharmonic Debye model, although the model has been applied successfully to the investigations of the thermodynamics properties of many materials (Bouhemadou et al 2011; Sun et al 2011).

\subsection{High pressure phase transition}

From the drawn Gibbs free energy with the help of the quais-harmonic Debye model, it is difficult to calculate and discuss the phase transition of one crystal material. This is pointed out in $\S 3.1$. So the investigations for the high pressure phase transitions of CdSe were carried out using Broyden-Fletcher-Goldfarb-Shenno (BFGS) minimization technique (Pfrommer et al 1997) implemented in CASTEP code.

Table 1. Lattice constants $a$ and $c(\AA)$, primitive cell volume $V_{0}\left(\AA^{3}\right)$, bulk modulus $B_{0}$ and its pressure derivative $B_{0}^{\prime}$ for $\mathrm{ZB}$ and $\mathrm{WZ}$ structural $\mathrm{CdSe}$ at $0 \mathrm{GPa}$ and $0 \mathrm{~K}$.

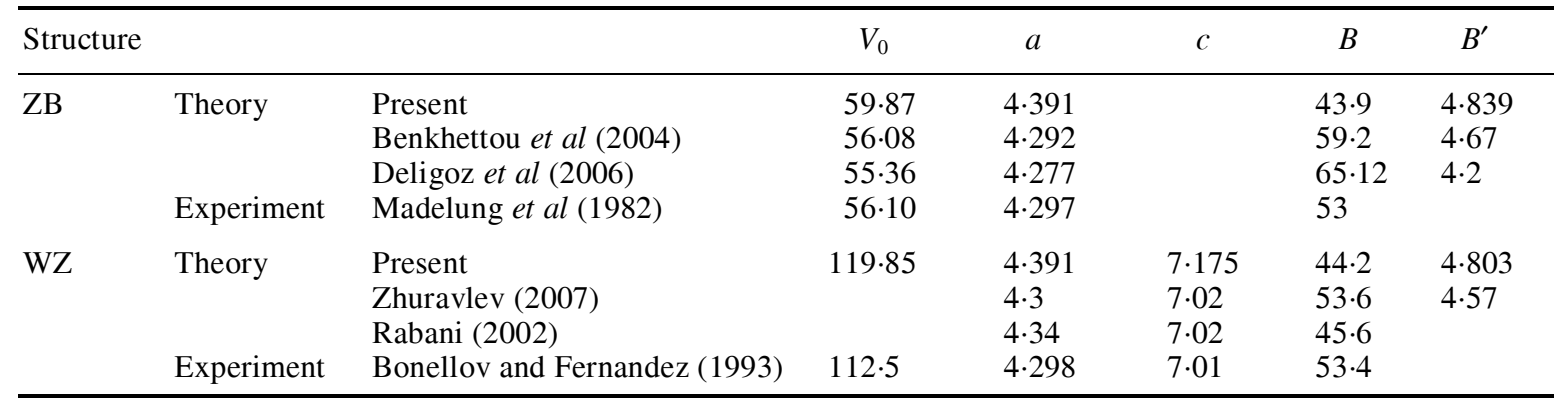


Table 2. Transition pressure $P_{\mathrm{t}}(\mathrm{GPa})$ and the corresponding volume collapses at $0 \mathrm{~K}$ for CdSe.

\begin{tabular}{|c|c|c|c|c|c|c|}
\hline \multirow[b]{3}{*}{ Structural transitions } & \multicolumn{3}{|c|}{ Transition pressure $P_{\mathrm{t}}$} & \multicolumn{3}{|c|}{ Volume collapses (\%) } \\
\hline & \multicolumn{2}{|r|}{ Theory } & \multirow[b]{2}{*}{ Experiment } & \multicolumn{2}{|r|}{ Theory } & \multirow[b]{2}{*}{ Experiment } \\
\hline & Present & Others & & Present & Others & \\
\hline $\mathrm{WZ} / \mathrm{ZB} \rightarrow \mathrm{Rs}$ & $3 \cdot 8$ & $\begin{array}{l}3 \cdot 5 \text { (Benkhettou et al 2004), } \\
2 \cdot 5 \text { (Cote } \text { et al } 1997)\end{array}$ & $\begin{array}{l}3 \text { (Yu and Gielisse, } \\
\text { 1971) }\end{array}$ & $19 \cdot 6$ & $\begin{array}{l}19 \cdot 8 \\
\text { (Cote et al 1997) }\end{array}$ & \\
\hline $\mathrm{Rs} \rightarrow \mathrm{Cmcm}$ & 29 & $\begin{array}{l}20 \text { (Benkhettou et al 2004), } \\
29 \text { (Cote } \text { et al 1997) }\end{array}$ & $\begin{array}{l}27 \text { (McMahon and } \\
\text { Nelmes 1996) }\end{array}$ & 1.4 & $1 \cdot 7$ & \\
\hline $\mathrm{Cmcm} \rightarrow \mathrm{CsCl}$ & 107 & & & $3 \cdot 6$ & (Cote et al 1997) & \\
\hline $\mathrm{Rs} \rightarrow \mathrm{CsCl}$ & 79 & $\begin{array}{l}95 \text { (Zakharov et al 1995) } \\
15 \text { (Benkhettou et al 2004) }\end{array}$ & & $5 \cdot 6$ & & \\
\hline
\end{tabular}

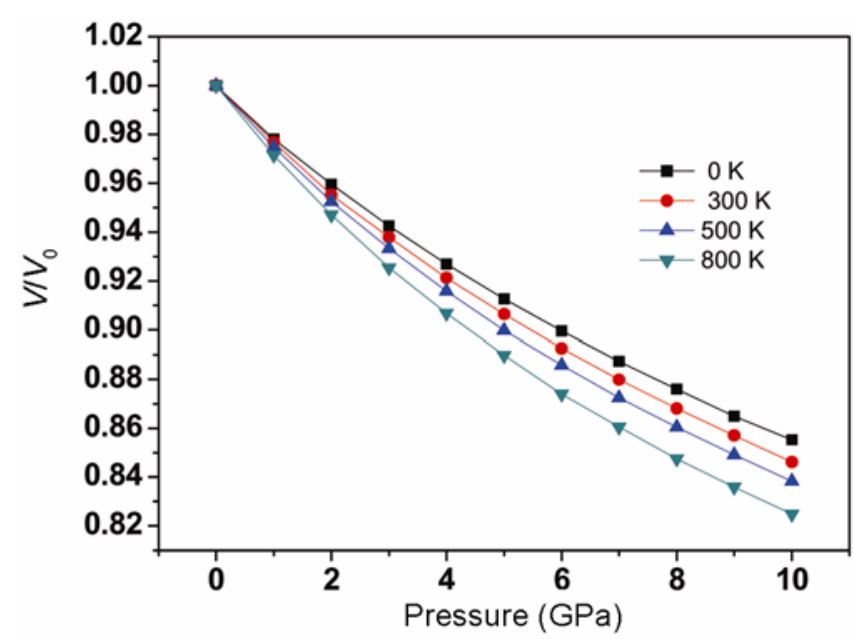

Figure 1. Thermal equations of state of $\mathrm{ZB}$ structural $\mathrm{CdSe}$ at $T=0,300,500$ and $800 \mathrm{~K}$.

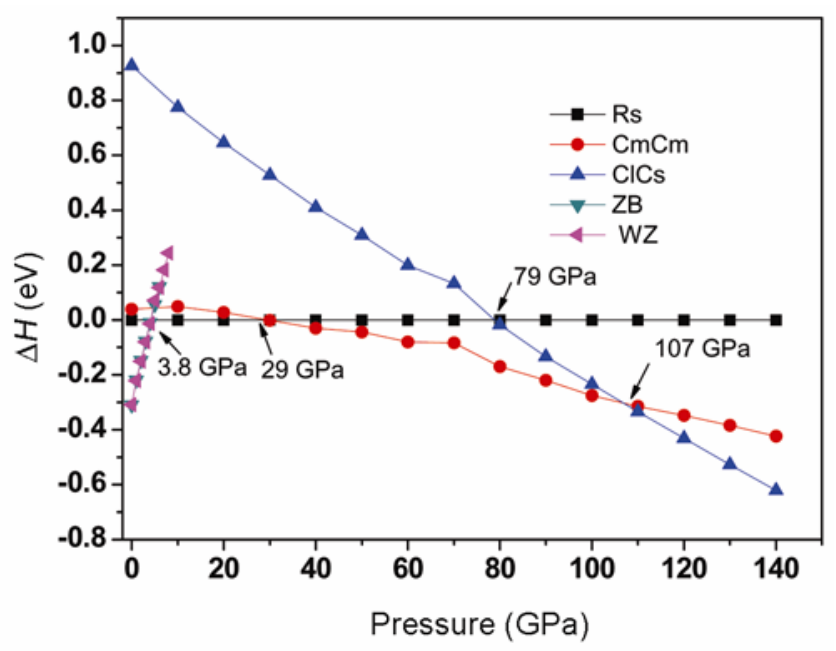

Figure 2. Enthalpy as a function of pressure for WZ, ZB, Rs, $\mathrm{Cmcm}, \mathrm{CsCl}$ structures taking $\mathrm{Rs}$ structure as reference for $\mathrm{CdSe}$ at $0 \mathrm{GPa}$ and $0 \mathrm{~K}$.
The intersections of the enthalpy-pressure curves of different structures in figure 2 show the transition pressures from one structure to the other structure and the stable ranges of the different structures. It is seen that the enthalpies of the $\mathrm{WZ}$ and $\mathrm{ZB}$ structural CdSe are almost same in the pressure range from 0 to $6 \mathrm{GPa}$. This corresponds to the fact that CdSe can crystallize in the $\mathrm{WZ}$ or ZB structure at ambient conditions. At about $3.8 \mathrm{GPa}$, the WZ/ZB structure transforms to the Rs structure. With continuous increasing pressure, the Rs structure transforms to the $\mathrm{Cmcm}$ structure at about $29 \mathrm{GPa}$. At the pressure, the optimized structural parameters for the $\mathrm{Cmcm}$ structure are: $\mathrm{Cd}: 4 c$ 0, 0.691, 0.25; Se: $4 c: 0,0 \cdot 198,0 \cdot 25$ with $a=5 \cdot 245, b=5 \cdot 328$ and $c=5 \cdot 135 \AA$. The $C m c m$ structure is stable in the pressure range from 29 to $107 \mathrm{GPa}$. After $107 \mathrm{GPa}$, the $\mathrm{CsCl}$ structure is more stable than the $\mathrm{Cmcm}$ structure. These transition pressures as well as the corresponding changes of volume collapses at transition points are listed in table 2 together with the available experimental and other theoretical data. It is seen that the present theoretical transition pressures from the WZ/ZB structure to the Rs structure and from the Rs structure to the $\mathrm{Cmcm}$ structure are overestimated by about 0.8 and $2 \mathrm{GPa}$ respectively, in contrast to the experimental data (Yu and Gielisse 1971; McMahon and Nelmes 1996). The deviations may originate in the above mentioned GGA calculations. From the deviations, it can be speculated that the drawn transition pressures from the RS structure to the $\mathrm{CsCl}$ structure or from the $\mathrm{Cmcm}$ structure to the $\mathrm{CsCl}$ structure also should be overestimated. The transition pressure from the $\mathrm{Cmcm}$ structure to the $\mathrm{CsCl}$ structure may be lower than $100 \mathrm{GPa}$. The overestimated transition pressures and the above overestimated lattice constants show the limitations of the theoretical techniques used in this work. However, the theoretical transition pressure from the RS structure to the $\mathrm{CsCl}$ structure is lower than the theoretical result obtained by Zakharov et al (1995). So, the present theoretical predictions may be more reasonable than those of 
Zakharov et al (1995). Combining with the above contrast between the theory and the experiments, the deviations should be in an appropriate range. In addition, the following several points need to be emphasized: (i) the present theoretical transition pressure from $\mathrm{RS}$ to $\mathrm{CsCl}$ is near to the result of Zakharov et al (1995), while Benkhettou et al (2004) underestimated the transition pressure, further obtaining the unreasonable phase transition sequence and the inappropriate stable ranges of phases (Benkhettou et al 2004); (ii) the Cmcm structure is the intermediate state from $\mathrm{Rs}$ to $\mathrm{CsCl}$, and a reasonable pressure-induced phase transition sequence $\mathrm{WZ} / \mathrm{ZB} \rightarrow \mathrm{Rs} \rightarrow \mathrm{Cmcm} \rightarrow$ $\mathrm{CsCl}$ is drawn. In an experiment, Nelmes and McMahon (1998) carried out an ADX study of CdSe up to $85 \mathrm{GPa}$ and found the evidence of a further transition from the $\mathrm{Cmcm}$ structure to a distorted- $\mathrm{Cmcm}$ structure at $36 \mathrm{GPa}$. This distorted- $\mathrm{Cmcm}$ structure has so far remained unsolved despite considerable experimental efforts, while the present stable range (29-107 GPa) for the $\mathrm{Cmcm}$ structure is much larger than the observed stable range (27-36 GPa). So, there should be certain intermediate states between the $\mathrm{Cmcm}$ structure and the $\mathrm{CsCl}$ structure and (iii) the change of volume collapse at the transition from Rs to $\mathrm{Cmcm}$ is very small and the structural transition might belong to the second-order phase transition, while other transitions should attribute to the first-order phase transitions.

\section{Conclusions}

In the short communication, the pressure-induced phase transition sequence $\mathrm{WZ} / \mathrm{ZB} \rightarrow \mathrm{Rs} \rightarrow \mathrm{Cmcm} \rightarrow \mathrm{CsCl}$ for $\mathrm{CdSe}$ was drawn reasonably for the first time. The corresponding transition pressures are 3.8, 29 and $107 \mathrm{GPa}$, respectively. The transition $\mathrm{Cmcm} \rightarrow \mathrm{CsCl}$ was predicted appropriately. Combining with the experimental data, it was speculated that there should be the intermediate states between the $\mathrm{Cmcm}$ structure and the $\mathrm{CsCl}$ structure. This may provide a reference for the future experimental and theoretical studies.

\section{Acknowledgements}

The authors thank the support by the Science-Technology Foundation of Guizhou Province (Grant No. [2013]2240), and the Science Foundation of Education Bureau of Guizhou Province, China (Grant No. KY(2012)051 and Grant No. 2010053), and Natural Science Research Project QJTD[2013]16 administrated by Department of Education of Guizhou Province, as well as by the support of Doctor Research Fund of Guizhou Normal College (Project No. 12BS021).

\section{References}

Asami H, Abe Y, Ohtsu T, Kamiya I and Hara M 2003 J. Phys. Chem. B107 12566
Benkhettou N, Rached D, Soudini B and Driz M 2004 Phys. Status Solidi (b) 1101

Bhuse V M 2005 Mater. Chem. Phys. 9160

Birch F 1947 Phys. Rev. 71809

Blanco A M, Francisco E and Luana V 2004 Comput. Phys. Commun. 15857

Bonello B and Fernandez B 1993 J. Phys. Chem. Solids 54209

Bouhemadou A, Zerarga F, Almuhayya A and Bin-Omran S 2011 Mater. Res. Bull. 122252

Chahboun A, Levichev S, Rolo A G, Conde O and Gomes M J M 2009 J. Luminescence 1291235

Chen C C, Chiu M Y, Sheu J T and Wei K H 2008 Appl. Phys. Lett. 92143105

Cote M, Zakharov O, Rubio A and Cohen M L 1997 Phys. Rev. B55 13025

Deligoz E, Colakoglu K and Ciftci Y 2006 Physica B373 124

Hankare P P, Bhuse V M, Garadkar K M, Delekar S D and Mulla I S 2004 Semicond. Sci. Technol. 1970

He C Y, Gao C X, Ma Y Y, Liu B G, Li M, Huang X W, Hao A M, Yu C L, Zhang D M, Liu H W and Zou G T 2008 J. Phys. Chem. Solids 69227

Hodes G, Yaron A A, Decker F and Motisuke P 1987 Phys. Rev. B36 4215

Huang M Z and Ching W Y1993 Phys. Rev. B47 9449

Madelung O, Schulz M, Weiss H and Landolt-Börstein (eds) 1982 Numerical data and functional relationships in science and technology (Berlin: Springer) Vol. 17

McMahon M I and Nelmes R J 1996 Phys. Status Solidi (b) 198 389

Milman V, Winkler B, White J A, Packard C J, Payne M C, Akhmatskaya E V and Nobes R H 2000 Int. J. Quantum Chem. 77895

Mohamed S H and Ali H M 2011 J. Appl. Phys. 109013108

Nelmes R J and McMahon M I 1998 In High pressure in semiconductor physics (eds) T Suski and W Paul (New York: Academic Press)

Ouendadji S, Ghemid S, Meradji H and El Haj Hassan F 2011 Comp. Mater. Sci. 501460

Payne M C, Teter M P, Allen D C, Arias T A and Joannopoulos J D 1992 Rev. Mod. Phys. 641045

Perdew J P, Burke K and Ernzerhof M 1996 Phys. Rev. Lett. 77 3865

Pfrommer B G, Côté M, Louie S G and Cohen M L 1997 J. Comp. Physiol. 131233

Rabani E 2002 J. Phys. Chem. 116258

Ramaiah K S, Sua Y K, Chang S J, Juang F S, Ohdairac K, Shiraki Y, Liu H P, Chen L G and Bhatnagar A K 2001 J. Cryst. Growth 22474

Sarasamak K, Limpijumnong S and Lambrecht W R L 2010 Phy. Rev. B82 035201

Sun X W, Song T, Liu Z J, Zhang C R, Tian J H and Guo P 2011 Solid State Commn. 211507

Vanderbilt D 1990 Phys. Rev. B41 7892

Xu Y N and Ching W Y 1993 Phys. Rev. B48 4335

Yu W C and Gielisse P J 1971 Mater. Res. Bull. 6621

Zakharov O, Rubio A and Cohen M L 1995 Phys. Rev. B51 4926

Zaoui A and Elhaj Hassan F 2001 J. Phys: Condens. Matter 13 253

Zhuravlev K K 2007 Physica B394 1 